\title{
Sensitivity of Dynamic Stability to Changes in Step Width During Treadmill Walking by Young Adults
}

\author{
Noah J. Rosenblatt, Christopher P. Hurt, and Mark D. Grabiner \\ University of Illinois at Chicago
}

\begin{abstract}
Recent experimental findings support theoretical predictions that across walking conditions the motor system chooses foot placement to achieve a constant minimum "margin of stability" $\left(\mathrm{MOS}_{\min }\right)$-distance between the extrapolated center of mass and base of support. For example, while step width varies, similar average MOS $_{\text {min }}$ exists between overground and treadmill walking and between overground and compliant/irregular surface walking. However, predictions regarding the invariance of $\mathrm{MOS}_{\min }$ to step-by-step changes in foot placement cannot be verified by average values. The purpose of this study was to determine average changes in, and the sensitivity of $\mathrm{MOS}_{\min }$ to varying step widths during two walking tasks. Eight young subjects walked on a dual-belt treadmill before and after receiving information that stepping on the physical gap between the belts causes no adverse effects. Information decreased step width by $17 \%(p=.01)$, whereas $\operatorname{MOS}_{\min }$ was unaffected $(p=.12)$. Regardless of information, subject-specific regressions between step-by-step values of step width and $\mathrm{MOS}_{\min }$ explained, on average, only $5 \%$ of the shared variance $(\beta=0.11 \pm 0.05)$. Thus, $\mathrm{MOS}_{\min }$ appears to be insensitive to changing step width. Accordingly, during treadmill walking, step width is chosen to maintain $\mathrm{MOS}_{\text {min }}$. If $\mathrm{MOS}_{\text {min }}$ remains insensitive to step width across other dynamic tasks, then assessing an individual's stability while performing theses tasks could help describe the health of the motor system.
\end{abstract}

Keywords: mediolateral, balance, gait

Proper foot placement during gait, as quantified by step width, is thought to be important in the step-by-step maintenance of frontal plane stability (Bauby \& Kuo, 2000; Hurt et al., 2010; Townsend, 1985). Accordingly, the wider steps, reportedly taken by older adults (Schrager et al., 2008) may increase lateral stability by increasing the distance between the lateral edge of the foot and the center of mass. However, this view may be overly simplistic. Inverted pendulum-based models considering only center of mass position are poorer predictors of dynamic behaviors, including forward motion during gait termination and the need for compensatory steps after external perturbations, compared with models including position and velocity (states) of the center of mass (Pai \& Patton, 1997; Pai et al., 1998). Using these states, a recent model defines the "margin of stability" as the distance between the base of support and extrapolated center of mass (XCOM; see Eq. 1) (Hof, 2008).

In contrast to other models describing stability during gait (Townsend 1985; Redfern \& Schumann, 1994; Pai \& Patton, 1997), margin of stability involves minimal computation and can quantitatively assess stability for a single given step. Further it may provide

Noah J. Rosenblatt (Corresponding Author), Christopher P. Hurt, and Mark D. Grabiner are with the Department of Kinesiology and Nutrition, University of Illinois at Chicago, Chicago, IL. additional information than other measures regarding the systems' dynamic state. For example, while, for young adults, local dynamic stability differs between treadmill and overground walking (Dingwell et al., $2001)$, the average minimum margin of stability $\left(\mathrm{MOS}_{\min }\right)$ remains constant in spite of the $\sim 20 \%$ increase in step width during treadmill walking (Rosenblatt \& Grabiner, 2010). The invariance of margin of stability, even when average step width is altered, is generally consistent with theoretical predictions of the measure (Hof, 2008). However, theoretical predictions relate to step-by-step behavior whereas the previous investigations compared mean values of $\operatorname{MOS}_{\min }$ across conditions. It is unclear whether, across dynamic tasks, $\operatorname{MOS}_{\min }$ is insensitive to naturally occurring step-by-step variations in step width. Such insensitivity would further validate the model and support that $\mathrm{MOS}_{\min }$ is a variable of interest to the motor system.

The purpose of this study was to determine average changes in, and the sensitivity of $\mathrm{MOS}_{\min }$ to varying step widths during two walking tasks. In the current study, step width was unobtrusively manipulated during treadmill walking and $\mathrm{MOS}_{\min }$ was measured before and after manipulation. We hypothesized that on average, dynamic stability $\left(\mathrm{MOS}_{\min }\right)$ would be invariant to changes in step width and, further, it would be insensitive to step-by-step changes in step width during each condition. 


\section{Methods}

Eight healthy young subjects (age: $21.6 \pm 0.9$ years, height: $169.6 \pm 6.8 \mathrm{~cm}$, mass: $64.3 \pm 9.8 \mathrm{~kg}$ ), provided written informed consent to participate in this institutionally approved study. Subjects performed two 10-min walking trials on a dual-belt treadmill (Active Step, Simbex, Lebanon, NH) (Figure 1), each performed at 1.2 $\mathrm{m} / \mathrm{s}$, the approximate preferred walking speed for healthy young adults (Himann et al., 1988).

For the first trial, the control condition, subjects received no information regarding dual-belt treadmill walking. Before the second trial, the information condition, subjects were explicitly informed that stepping on the $1.5 \mathrm{~cm}$ physical gap between the treadmill belts has no adverse effects. Previous observations suggest that in absence of information, subjects appear to avoid stepping on the between-belt gap. Consequently, we reasoned that imparting information would easily, unobtrusively and effectively influence foot placement, and step width.

The positions of 22 reflective markers placed over body landmarks (Kadaba et al., 1990) were tracked using a motion capture system operating at $60 \mathrm{~Hz}$ (Motion Analysis, Santa Rosa, CA). Using marker positions, a 10-segment rigid body model was constructed from which step width and $\mathrm{MOS}_{\min }$ were computed (Matlab, Mathworks, Natick, MA). Step width was calculated as the distance in the frontal plane between the estimated foot centroids sampled at successive midstances (Figure 1).

The position of the center of mass of each model segment was estimated using anthropometric data (Winter, 2005). These were weighted by segmental masses, filtered using a fourth-order Butterworth filter with $12 \mathrm{~Hz}$ cutoff, and averaged to provide the whole body center of mass position (COM in Eq 1). A first-central difference algorithm computed center of mass velocity $\left(\mathrm{COM}_{\mathrm{vel}}\right)$ and extrapolated center of mass (XCOM) was calculated as follows:

$$
\mathrm{XCOM}=\mathrm{COM}+\mathrm{COM}_{\mathrm{vel}} / \omega_{0}
$$

where $\omega_{0}$ is the natural frequency of a noninverted pendulum with length 1.34 times trochanteric height (Hof, 2008). The lateral border of the base of support $\left(\mathrm{BOS}_{\mathrm{lat}}\right)$ was quantified using previously described methods (Rosenblatt \& Grabiner, 2010) and the margin of stability (MOS) was defined as follows:

$$
\mathrm{MOS}=\mathrm{BOS}_{\text {lat }}-\mathrm{XCOM}
$$

MOS was calculated from heel strike to contralateral toeoff, and the minimum $\left(\mathrm{MOS}_{\mathrm{min}}\right)$ was extracted. Larger positive values of $\mathrm{MOS}_{\min }$ represent increased stability during the measurement period. Step time, which theoretically influences $\mathrm{MOS}_{\min }$ (Hof 2008), was calculated as the time between consecutive heelstrikes.

Habituation to walking on a treadmill may result in time-dependent trends in movement patterns (Wall \& Charters, 1980). With regard to this study, these trends could manifest in measures of step width and $\mathrm{MOS}_{\min }$ during the control condition, which was necessarily performed first. To test for time-dependent trends in step width and $\mathrm{MOS}_{\min }$, linear regressions were performed between step width and step number during the control
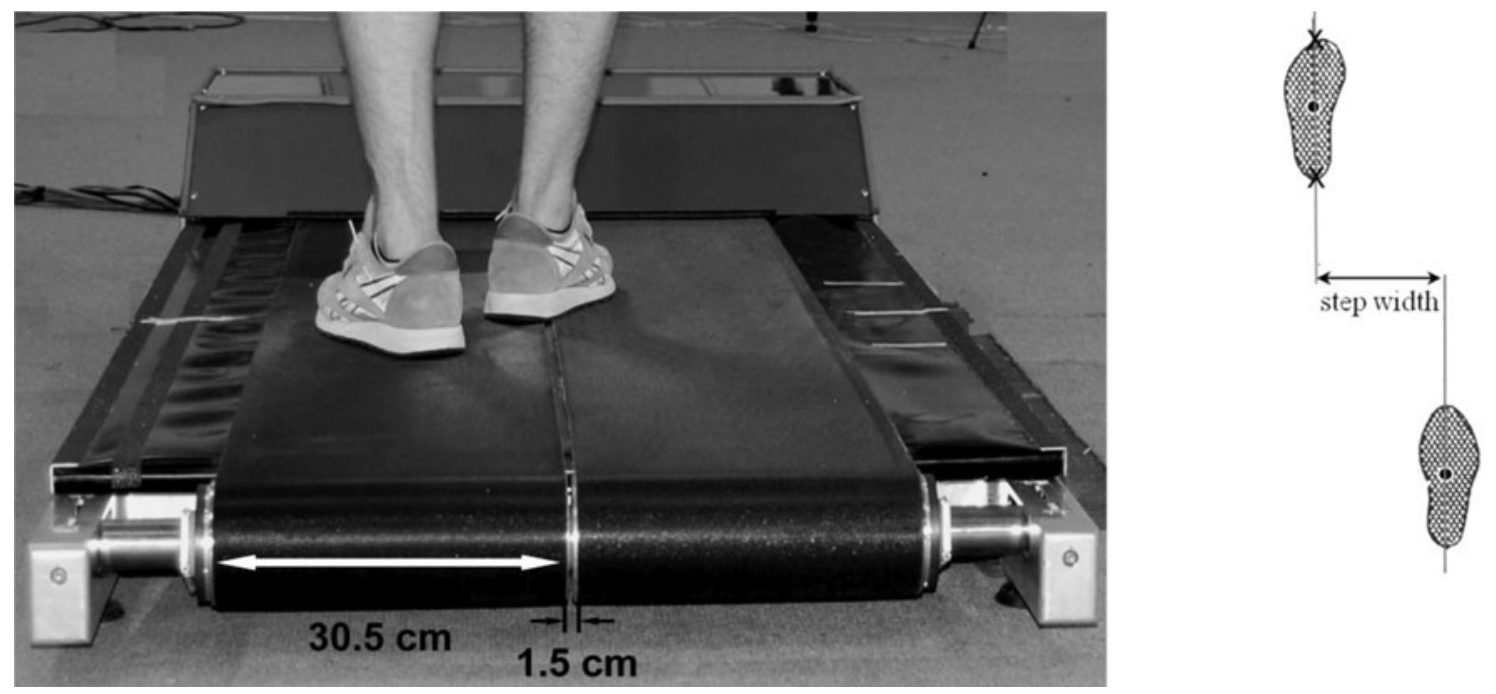

Figure 1 - Split-belt treadmill design and definition of step width (left). Each treadmill belt of the split-belt treadmill is $30.5 \mathrm{~cm}$ wide, and the two are separated by a physical gap that is $1.5 \mathrm{~cm}$ wide, allowing the foot to easily span the gap on any given step. Right: The foot centroid was estimated as the midpoint (filled black circle) of the line (light gray) connecting reflective markers on the heel and metatarsal (each bold "X"). The distance in the frontal plane between foot centroids at successive midstances defined step width. Midstance was the point when the lateral malleolus of the swing limb passed anterior to that of the stance limb, and corresponded to when the base of support (footprints) was stationary. The schematic representation of the feet does not correspond with the foot placement in the photograph. 
condition, and between $\mathrm{MOS}_{\min }$ and step number during the control condition. Significant regressions with $R^{2} \geq 0.25$ were considered meaningful (Vincent 1999). For these cases it was desirable to include in the group analysis only those points for which the time-dependent trend was absent.

To determine the maximum number of data points for which a time-dependent trend was absent, a linear
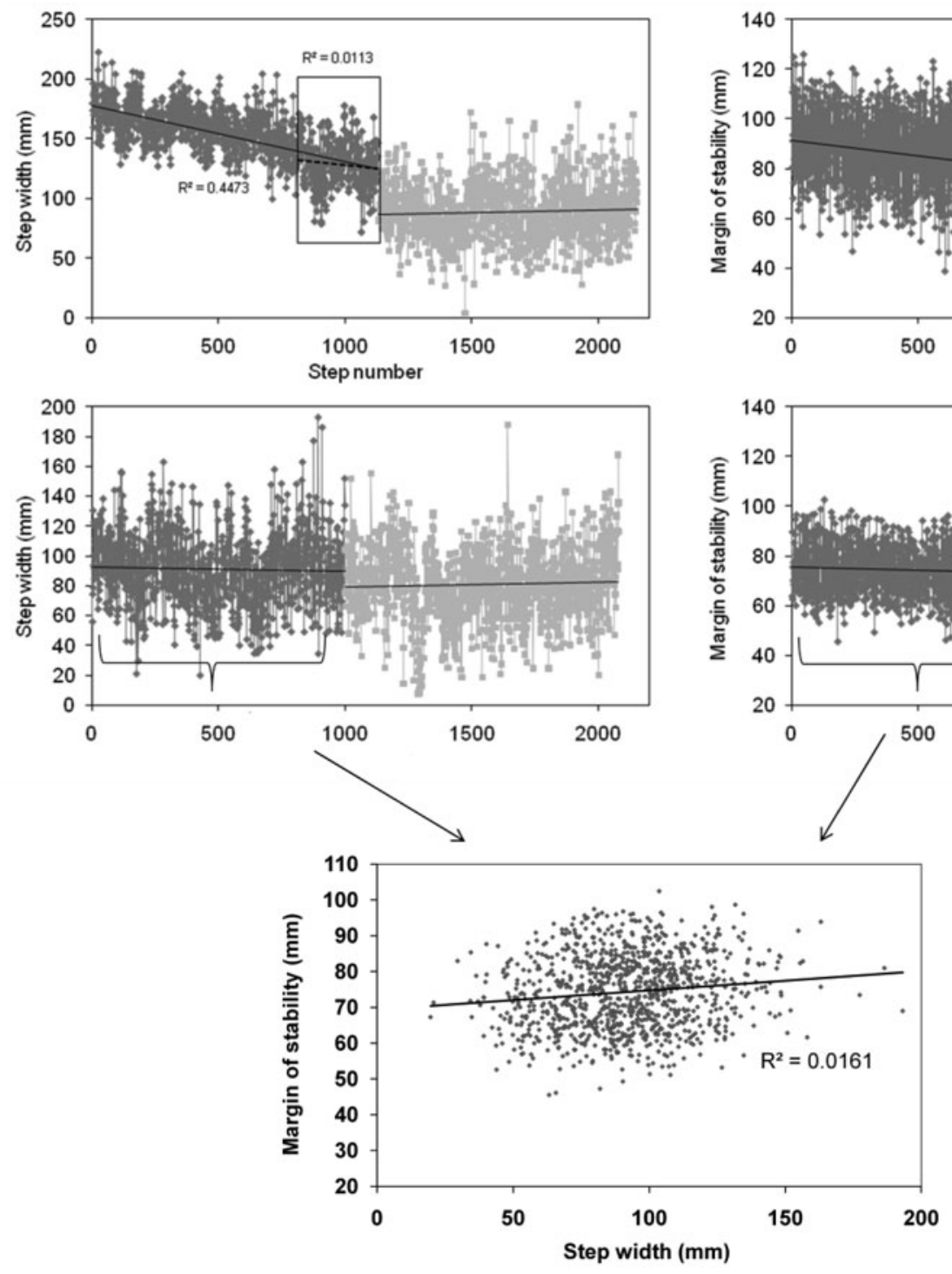

Figure 2 - Step width and minimum margin of stability $\left(\mathrm{MOS}_{\mathrm{min}}\right)$ across conditions. Regressions between step width and step number (two left-most panels) and minimum margin of stability $\left(\mathrm{MOS}_{\min }\right.$ ) and step number (two right-most panels) during the control condition (dark gray data points) for two subjects. Data, and best-fit line for the information condition (light gray) shown, although not subjected to the same analysis as control condition data. Top, left-most panel: Data from one of two subjects for whom a meaningful relationship between all step widths and step numbers was found $\left(R^{2}=.44\right)$. The 317 data points included in the box represent the maximum number of data points for which no time-dependent relationship existed. The best-fit regression line for this data (dotted black) was not significnat $\left(R^{2}=.01 ; p=.058\right)$. Top, right-most panel: For this subject the relationship between $\mathrm{MOS}_{\min }$ and step number during the control condition was not meaningful $\left(R^{2}=.05\right)$. Two middle panels: Exemplary data of a subject for whom the relationship between all step widths and step numbers during the control condition, and the relationship between all $\mathrm{MOS}_{\min }$ and step numbers during the control condition was not significant. Bottom-most panel: Linear regression bewteen individual values of step width and $\mathrm{MOS}_{\min }$ for the control condition for the same subject shown in the middle two panels. Only $2 \%$ of the variability in $\mathrm{MOS}_{\min }$ was explained by the relationship. Data are exemplary of all subejcts for all conditions (see Table 1). 
(Figure 2). The same points were analyzed for step width and $\mathrm{MOS}_{\mathrm{min}}$.

A paired $t$ test was used to first determine if the between-condition difference in subject-averaged step widths was significant. If so, then a paired $t$ test was used to determine if the between-condition differences in subject-averaged $\mathrm{MOS}_{\min }$ and step time were significant. To understand the sensitivity of $\mathrm{MOS}_{\min }$ to step width, across each subject and condition, a linear regression was fit to all included step widths and corresponding $\mathrm{MOS}_{\text {min }}$ (Figure 2). The resulting regression coefficient ( $\beta$ ) describes the sensitivity of $\mathrm{MOS}_{\min }$ to step width. Significant regressions with $R^{2} \geq .25$ were considered meaningful. Analyses were done using SPSS software (SPSS Inc, Chicago, IL), with significance given for $p<.05$.

\section{Results}

No time dependent trend was detected in $\mathrm{MOS}_{\min }$. With respect to step width, a meaningful linear relationship was shown for two subjects, for whom $27 \%$ and $44 \%$ of the variance in step width was explained by the number of steps. Significant relationships were absent in the final 296 and 317 steps of the control condition, respectively. These steps were submitted to group analysis. A significant regression was found for three other subjects, although the average amount of variance explained was $<7 \%$.

Compared with the control condition, step width was narrower in the information condition. Providing information regarding the between-belt gap was associated with a $17 \%$ reduction of step width $(119 \pm 40 \mathrm{~mm}$ vs. 99 $\pm 34 \mathrm{~mm} ; p=.01)$. There was only a $6 \%$ difference in $\mathrm{MOS}_{\mathrm{min}}$ between control and information conditions $(70 \pm$ $14 \mathrm{~mm}$ vs. $66 \pm 10 \mathrm{~mm}$, respectively; $p=.12$ ) (Figure 3).

At the individual level, on a step-by-step basis, changes in step width were unrelated to changes in $\mathrm{MOS}_{\text {min }}$ (Table 1). While step width and $\mathrm{MOS}_{\text {min }}$ were significantly correlated for six subjects during the control condition, and seven during the information condition, at most these relationships accounted for $11 \%$ of the shared variance. Average $\beta$ values for the control and information condition were $0.11 \pm .06$ and $0.10 \pm 0.04$, respectively. Step time was similar between conditions $(522 \pm 38 \mathrm{~ms}$ vs. $522 \pm 33 \mathrm{~ms} ; p=.97)$.

\section{Discussion}

The purpose of this study was to determine average changes in, and the sensitivity of $\mathrm{MOS}_{\min }$ to varying step widths during two walking tasks. While step width was significantly narrower after providing information regarding the effects of stepping on the physical gap between the treadmill belts, on average, MOS $_{\text {min }}$ was not significantly affected. In addition, on a step-by-step basis, average regression coefficients suggest for every centimeter increase in step width, $\mathrm{MOS}_{\min }$ increases by approximately one millimeter, further supporting the hypothesis that $\mathrm{MOS}_{\mathrm{min}}$ would be insensitive to step width.

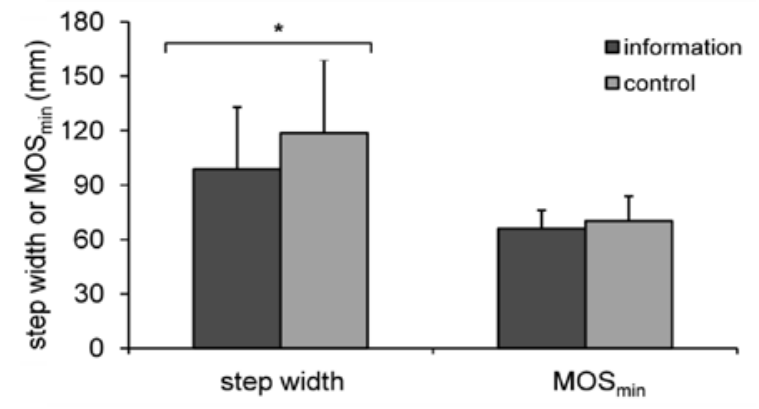

Figure 3 - Group average values for step width and minimum margin of stability during each condition. Mean values and standard deviations for step width and $\mathrm{MOS}_{\min }$ across condition, averaged across all subjects. *Significant differences using a paired $t$ test.

Table 1 Subject-specific values of regression coefficients and corresponding $R^{2}$ values describing the relationship between step width and minimum margin of stability during each condition

\begin{tabular}{lcccc}
\hline Subject & $\begin{array}{c}\boldsymbol{\beta} \\
\text { (Control) }\end{array}$ & $\begin{array}{c}\boldsymbol{R}^{\mathbf{2}} \\
\text { (Control) }\end{array}$ & $\begin{array}{c}\boldsymbol{\beta} \\
\text { (Information) }\end{array}$ & $\begin{array}{c}\boldsymbol{R}^{\mathbf{2}} \\
\text { (Information) }\end{array}$ \\
\hline 1 & 0.05 & 0.02 & 0.05 & 0.02 \\
2 & - & - & 0.12 & 0.03 \\
3 & 0.06 & 0.02 & 0.03 & 0.01 \\
4 & 0.12 & 0.07 & 0.14 & 0.14 \\
5 & 0.22 & 0.05 & 0.14 & 0.06 \\
6 & - & - & - & - \\
7 & 0.09 & 0.04 & 0.08 & 0.04 \\
8 & 0.15 & 0.11 & 0.13 & 0.06 \\
\hline
\end{tabular}

Note. Values for the regression coefficient $(\beta)$ and $R^{2}$ are listed for only those cases where a significant relationship $(p<0.05)$ was found. 
The insensitivity of $\mathrm{MOS}_{\min }$ to changes in step width suggests that during gait the motor system may adjust step width to maintain a constant $\mathrm{MOS}_{\min }$, which is consistent with theoretical expectations (Hof, 2008). Attaining a stable gait by adjusting foot placement based on the states of the center of mass at foot contact has long been described (Townsend, 1985). Although this work and similar ensuing studies (MacKinnon \& Winter, 1993; Redfern \& Schumann, 1994) provide insight into control laws or kinetic constraints governing mediolateral stability, none directly quantify stability. Measures including local dynamic stability (Dingwell et al., 2001) or the feasible limits of stability (Pai \& Patton, 1997) can in fact quantify stability. However, the former may not apply to step-by-step analyses and both use fairly complex computational methods. Moreover, $\mathrm{MOS}_{\min }$ can be simply and mechanistically interpreted; $\mathrm{MOS}_{\min }$ is "proportional to the minimum impulse needed to unbalance a subject" (Hof et al., 2005).

While this study validated model predictions during treadmill walking, predictions likely persist to other dynamics tasks, during which $\mathrm{MOS}_{\min }$ should continue to remain insensitive to step width. Thus, while an invariance of $\mathrm{MOS}_{\min }$ to changes in step width during treadmill and overground walking (Rosenblatt \& Grabiner, 2010), could possibly reflect differences in walking modes, it more likely reflects control of $\mathrm{MOS}_{\text {min }}$ during each conditions. Regarding overground walking, for example (Stolze et al., 1997), the changes in step width that result from stepping on a compliant or irregular surface, also result in margins of stability similar to those while stepping on noncompliant and smooth surfaces, respectively (MacLellan \& Patla, 2006; Curtze et al., 2011). Regarding treadmill walking, for example, in response to small lateral perturbations, changes in foot placement are made such that the margin of stability is similar to that during unperturbed walking (Hof et al., 2010). If healthy, robust systems maintain a relatively constant $\mathrm{MOS}_{\min }$ across dynamic situations, then assessing the extent of such invariance may provide insight into the presence or degree of impaired balance.

Although not directly related to the study purpose, several points regarding treadmill walking are noteworthy. Given that simply informing subjects about the effects of stepping on the gap resulted in decreased step width suggests subjects may have been avoiding the gap during the control condition. This is consistent with findings that step width increases when subjects walk with each foot on one side of a center guideline (Chow et al., 2009). Accordingly, treadmill characteristics such as the physical gap have the potential to alter kinematics. It may therefore be useful to consider the influence of treadmill characteristics, e.g., single vs. dual-belt design or treadmill-belt width, when designing or comparing treadmill walking studies. This warrants further investigation. In addition, for some healthy, young subjects-likely those naïve to treadmill walking-it may be necessary to account for habituation when analyzing step width or margin of stability, by considering step-by-step behavior.
In general, when a large number of steps are available, step-by-step analysis could provide more insight than mean values alone (Hurt et al., 2010).

In summary, step width on a dual-belt treadmill significantly decreased after subjects were informed that stepping on the between-belt gap had no negative conse-

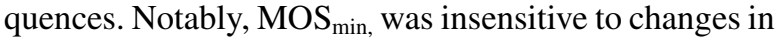
step width, providing additional evidence that the goal of foot placement is to attain a particular $\operatorname{MOS}_{\min }$.

\section{References}

Bauby, C.E., \& Kuo, A.D. (2000). Active control of lateral balance in human walking. Journal of Biomechanics, 33, 1433-1440. PubMed doi:10.1016/S00219290(00)00101-9

Chow, J.W., Hemleben, M.E., \& Dobrivoje, S.S. (2009). Effect of center-line guided walking on gait characteristics in healthy subjects. Journal of Biomechanics, 42, 1134-1137. PubMed doi:10.1016/j.jbiomech.2009.02.024

Curtze, C., Hof, A., Postema, K., \& Otten, B. (2011). Over rough and smooth: Amputee gait on an irregular surface. Gait \& Posture, 33, 292-296. PubMed doi:10.1016/j. gaitpost.2010.11.023

Dingwell, J.B., Cusumano, J.P., Cavanagh, P.R., \& Sternad, D. (2001). Local dynamic stability versus kinematic variability of continuous overground and treadmill walking. Journal of Biomechanical Engineering, 123, 27-32. PubMed doi:10.1115/1.1336798

Himann, J.E., Cunningham, D.A., Rechnitzer, P.A., \& Paterson, D.H. (1988). Age-related changes in speed of walking. Medicine and Science in Sports and Exercise, 20, 161-166. PubMed doi:10.1249/00005768-198820020-00010

Hof, A.L. (2008). The 'extrapolated center of mass' concept suggests a simple control of balance in walking. Human Movement Science, 27, 112-125. PubMed doi:10.1016/j. humov.2007.08.003

Hof, A.L., Gazendam, M.G.J., \& Sinke, W.E. (2005). The condition of dynamic stability. Journal of Biomechanics, 38, 1-8. PubMed doi:10.1016/j.jbiomech.2004. 03.025

Hof, A.L., Vermerris, S.M., \& Gjaltema, W.A. (2010). Balance responses to lateral perturbations in human treadmill walking. The Journal of Experimental Biology, 213, 2655-2664. PubMed doi:10.1242/jeb.042572

Hurt, C.P., Rosenblatt, N., Crenshaw, J.P., \& Grabiner, M.D. (2010). Variation in trunk kinematics influences variation in step width during treadmill walking by older and younger adults. Gait \& Posture, 31, 461-464. PubMed doi:10.1016/j.gaitpost.2010.02.001

Kadaba, M.P., Ramakrishnan, H.K., \& Wootten, M.E. (1990). Measurement of lower extremity kinematics during level walking. Journal of Orthopaedic Research, 8, 383-392. PubMed doi:10.1002/jor.1100080310

MacKinnon, C.D., \& Winter, D.A. (1993). Control of whole body balance in the frontal plane during human walking. Journal of Biomechanics, 26, 633-644. PubMed doi:10.1016/0021-9290(93)90027-C

MacLellan, M.J., \& Patla, A.E. (2006). Adaptations of walking on a compliant surface to regulate dynamic stability. Experimental Brain Research, 173, 521-530. PubMed doi:10.1007/s00221-006-0399-5

Pai, Y-C., \& Patton, J. (1997). Center of mass velocityposition predictions for balance-control. Journal of 
Biomechanics, 30, 347-354. PubMed doi:10.1016/S00219290(96)00165-0

Pai, Y-C., Rogers, M.W., Patton, J., Cain, T.D., \& Hanke, T.A. (1998). Static versus dynamic predictions of protective stepping following waist perturbations in young and older adults. Journal of Biomechanics, 31, 1111-1118. PubMed doi:10.1016/S0021-9290(98)00124-9

Redfern, M.S., \& Schumann, T. (1994). A model of foot placement during gait. Journal of Biomechanics, 27, 1339-1346. PubMed doi:10.1016/0021-9290(94)90043-4

Rosenblatt, N.J., \& Grabiner, M.D. (2010). Measures of frontal plane stability during treadmill and overground walking. Gait \& Posture, 31, 380-384. PubMed doi:10.1016/j. gaitpost.2010.01.002

Schrager, M.A., Kelly, V.E., Price, R., Ferrucci, L., \& ShumwayCook, A. (2008). The effects of age on medio-lateral stability during normal and narrow base walking. Gait \& Posture, 28, 466-471. PubMed doi:10.1016/j.gaitpost.2008.02.009
Stolze, H., Kuhtz-Buschbeck, J.P., Mondwurf, C., BoczekFuncke, A., Jöhnk, K., Deuschel, G., \& Illert, M. (1997). Gait analysis during treadmill and overground locomotion in children and adults. Electroencephalography and Clinical Neurophysiology, 105, 490-497. PubMed doi:10.1016/ S0924-980X(97)00055-6

Townsend, M.A. (1985). Biped gait stabilization via foot placement. Journal of Biomechanics, 18, 21-38. PubMed doi:10.1016/0021-9290(85)90042-9

Vincent, W.J. (1999). Statistics in kinesiology. Champaign, IL: Human Kinetics.

Wall, J.C., \& Charters, J. (1980). The process of habituation to treadmill walking at different velocities. Ergonomics, 23, 425-435. PubMed doi:10.1080/00140138008924758

Winter, D.A. (2005). Anthropometry. In Biomechanics and Motor Control of Human Movement (pp. 59-86). Hoboken, NJ: John Wiley \& Sons, Inc. 